\title{
Determinants Of Adherence To Dietary Prescription Among Patients With End-Stage Renal Disease Undergoing Hemodialysis In Dar Es Salaam, Tanzania
}

Rahab Benjamin ( $\sim$ rahabbenny@gmail.com )

Aga Khan University

Tumbwene E. Mwansisya

Aga Khan University

Mary Lyimo

Aga Khan University

\section{Research Article}

Keywords: determinants, adherence, end stage renal disease, dietary prescription, hemodialysis

Posted Date: August 6th, 2021

DOI: https://doi.org/10.21203/rs.3.rs-729734/v1

License: (c) (1) This work is licensed under a Creative Commons Attribution 4.0 International License.

Read Full License 


\section{Abstract}

Background: The increasing prevalence of chronic kidney disease means it has become a leading cause of death worldwide and a global health concern. The prevalence of chronic kidney disease in Tanzania is $12.4 \%$. A major cause of mortality among patients with chronic kidney disease is non-adherence to dietary prescription. However, factors associated with non-adherence to dietary prescription remain unknown.

Methods: This study used a quantitative descriptive cross sectional design, To investigate determinants of adherence to dietary prescription among patients with end-stage renal disease undergoing hemodialysis in Dar es Salaam Region, Tanzania. Data were collected using a self-administered structured questionnaire.

Result: Data were obtained from 150 participants; $56.7 \%$ adhered to their prescribed diet. Factors significantly associated with adherence to dietary prescription were permanent residence in the city where the hospital was located $(p=0.018)$, age $(p=0.000)$, employment status $(p=0.017)$, duration of chronic kidney disease $(p=0.001)$, comorbidity $(p=0.029)$, family awareness $(p=0.003)$, affordability $(p=0.044)$ and convenience of nutritional counseling services $(p=0.046)$, and conversation with a healthcare provider regarding diet $(p=0.039)$. Predictors of adherence were permanent residence $(p=0.010)$, age $(p=0.002)$, duration of chronic kidney disease $(p=0.012)$, and conversation regarding diet with a healthcare provider $(p=0.018)$.

Conclusion These findings highlight a need for interventions to improve adherence to dietary prescription. Building understanding of determinants of adherence to dietary regimens among patients with end-stage renal disease is critical because of the poor progress associated with non-adherence. Further mixed method research should be conducted to gain an in-depth understanding of adherence to dietary prescription and associated factors.

\section{Introduction}

Chronic kidney disease (CKD) is a global health concern. The illness progresses to end-stage renal disease (ESRD), which is the advanced stage of CKD (1). Worldwide, CKD an accounted for an estimated 1.2 million deaths in 2005-2015, with most of these deaths in low- and middle-income countries (2). A systematic review conducted between 1995 and 2017 reported the prevalence of CKD in Africa was $10.1 \%$ (3). In sub Saharan Africa, the estimated CKD prevalence in the general population is $13.6 \%$ (4), that in East Africa is $14.4 \%$ (5), and that in rural Tanzania is $12.4 \%$ (6). However, the prevalence of CKD may vary with location. For example, Stanifer (7) reported a higher prevalence of CKD in urban compared with rural districts (15.2\% vs. $7.0 \%$ ) in Moshi municipality, in the Kilimanjaro Region of Tanzania.

Major causes of mortality among patients with ESRD are non-adherence to dietary prescription, hemodialysis sessions, and medication (8). Dietary modification is required to reduce the workload on kidney function and morbidity/mortality among patients on hemodialysis (9). Dietary recommendations 
aim to reduce further deterioration of kidney function, and require patients to limit food amounts to avoid accumulation of waste that cannot be excreted from the kidney (10). Complications associated with nonadherence to dietary prescription include bone demineralization, pulmonary edema, metabolic disorder, cardiovascular problems, and death (11). However, dietary adherence remains a challenge among patients with CKD, with a previous study reporting that $77 \%$ of patients deviated from their prescribed diet, predisposing them to further CKD complications (12).

In the African context, few studies have explored factors associated with dietary adherence. One study (13) reported factors associated with dietary adherence among adults with ESRD on hemodialysis were socioeconomic factors, condition and therapy factors, healthcare team- and system-related factors, patient-related factors, social gathering, and taste preferences. \{Lambert, 2017 \#30\} Moreover, a study from Kenya that investigated dietary adherence among patients with CKD reported $36.3 \%$ adhered to their dietary prescription (14). Reasons for non-adherence identified in that study were lack of flexibility of diet $(80 \%)$, difficulty in limiting fluid intake (41.1\%), and challenges in following the recommended diet (61.8\%). However, that study collected subjective information from patients to establish these adherence factors (14). In addition, previous studies were conducted in contexts that may differ from Tanzania. For example, Tanzanian patients are likely to attend traditional and modern treatment, with the potential for non-adherence to either treatment modality $(15,16)$.

The Tanzania Ministry of Health in collaboration with the private sector has invested in CKD management by training nephrology specialists, providing kidney transplant services, increasing the number of dialysis units, and reducing the expense of dialysis services (17). Despite these efforts, nutrition remains a challenge for patients with CKD. This is an important issue, as non-adherence to the prescribed diet may result in readmission and mortality due to metabolic disorder, anemia, pulmonary edema, or malnutrition. Dietary (including fluids) modification has been shown to have positive outcomes among patients with ESRD (18). Therefore, this study aimed to investigate the determinants of adherence to dietary prescription among patients with ESRD undergoing hemodialysis in Dar es Salaam, Tanzania.

\section{Methods}

\subsection{Study design and setting}

We used a quantitative approach with a descriptive cross sectional design to determine the prevalence of adherence to dietary prescription and describe relationships between associated factors. This study was conducted at the renal unit (hemodialysis unit) in Muhimbili National Hospital, which is a tertiary hospital providing inpatient services (1200 bed capacity) and multispecialty clinic outpatient services that receive up to 2000 patients per day. Dar es Salaam is a large city (former capital city) located in the coastal region of Tanzania. This hospital was selected because it is the national referral hospital and university teaching hospital that provides hemodialysis services for many patients with ESRD. Approximately 80100 patients (outpatients and inpatients) per day attend the nephrology department for hemodialysis. Renal transplant procedure or operations are usually done once every 2 months. 


\subsection{Study population and selection criteria}

The target population was patients with ESRD receiving hemodialysis; CKD accounts for around $80 \%$ of patients attending hemodialysis at the study hospital. All patients aged over 18 years that were diagnosed with ESRD (CKD stage 5) and had undergone hemodialysis for more than 1 month (i.e., attended the study hospital two or three times per week) were included in this study. Patients who were critically ill or unable to communicate were excluded at the time of data collection.

\subsection{Sample size}

The sample size of 150 patients was calculated using the Kish and Lislie formula (19). The proportion was derived from a study conducted in Kenya that found adherence to dietary prescription was $36.3 \%$ (Opiyo et al., 2019).

\subsection{Sampling technique}

Systemic random sampling was used to ensure participants were representative of the study population. The registration book in the hemodialysis unit showed 100 patients attended hemodialysis each day; around 60 were patients with CKD. We aimed to study 20 patients per day. The formula used to calculate the sampling interval was: $(k)=N / n$, where $K=60 / 20=3$. Therefore, the sampling interval was after every 3 patients.

\subsection{Data collection}

A self-administered paper-based questionnaire was used to collect data. This tool was adapted from a standardized questionnaire on dietary adherence and modified to address the objectives of this study based on a literature review (20). All questionnaire items were translated into Swahili as this is the common language used by participants. The questionnaire included closed-ended questions and covered major areas related to adherence to dietary recommendations e.g. in the past week, how many times did you follow dietary recommendation? Independent variables were demographic data, clinical characteristics, family support, and the healthcare system. The dependent variable was adherence to dietary prescription, which was measured by categorizing behaviors reported by participants. Patients who reported they adhered to dietary prescription "all of the time" or "most of the time" were classified as the adhered group and those who reported adherence "a few times," "very rarely," or "none of the time" formed the non-adhered group.

\subsection{Pre-testing of the data collection tool}


The questionnaire was tested for validity and reliability by conducting pilot study with 15 patients randomly selected from the hemodialysis unit. These participants were excluded at the time of actual data collection to avoid duplicated information. The Cronbach's alpha for the tool was 0.706 . The results from the pilot study were used to modify the questionnaire as necessary before actual data collection.

\subsection{Data collection and control}

Participants were given a 21 -item questionnaire that they were required to complete by themselves. The questionnaire took 20-30 minutes to complete, although some participants requested more time. Quality control was maintained by checking for completeness of the questionnaire or missing data. Data were stored securely in soft and hard copy, and only accessible to the researchers.

\subsection{Data analysis and interpretation}

Data were managed using SPSS version 25. The data were cleaned, coded, and then analyzed. Descriptive statistics (means, standard deviations, percentages, and frequencies) were used to describe sociodemographic data, participants' clinical characteristics, and the prevalence of adherence, and presented in tables. Inferential statistics were also calculated, with chi-square tests used to test the association between independent categorical variables and adherence. Logistic regression was then used to predict the relationships between independent variables and the dependent variable. The significance level was set at $5 \%$ or $p \leq 0.05$.

\section{Results}

\subsection{Participants' sociodemographic characteristics}

Of the 150 participants, $68.7 \%$ were male and $31.3 \%$ were female. The mean age was 48.4 , $\mathrm{SD} \pm 13.9$ years (range 18-77 years). More than half of the participants were married (66.7\%); 65.3\% lived in Dar es Salaam and 34.7\% lived upcountry i.e. outside Dar es Salaam region but with the boundary of Tanzania. The majority of participants were employed (including self-employed) (51.4\%), and most had a secondary school education. The rate of adherence to prescribed diets was 56.7\%. Other demographic characteristics are presented in Table 1.

Table 1: Participants' socio-demographic characteristics 


\begin{tabular}{|c|c|c|c|}
\hline Variable & & Frequency (n) & Percentage (\%) \\
\hline \multirow{3}{*}{ Age (years) } & $18-37$ & 36 & 24 \\
\hline & $38-57$ & 71 & 47.3 \\
\hline & $58-77$ & 43 & 28.7 \\
\hline \multirow[t]{2}{*}{ Permanent residence } & Dar es Salaam & 98 & 65.3 \\
\hline & Upcountry & 52 & 34.7 \\
\hline \multirow[t]{2}{*}{ Gender } & Male & 103 & 68.7 \\
\hline & Female & 47 & 31.3 \\
\hline \multirow[t]{5}{*}{ Marital status } & Single & 33 & 22.0 \\
\hline & Married & 100 & 66.7 \\
\hline & Divorced & 7 & 4.7 \\
\hline & Widowed & 8 & 5.3 \\
\hline & Cohabiting & 2 & 1.3 \\
\hline \multirow[t]{4}{*}{ Employment status } & Employed & 46 & 30.7 \\
\hline & Self-employed & 31 & 20.7 \\
\hline & Retired & 27 & 18.0 \\
\hline & Unemployed & 46 & 30.7 \\
\hline \multirow[t]{4}{*}{ Level of education } & Primary level & 44 & 29.3 \\
\hline & Secondary level & 60 & 40.0 \\
\hline & Collage/university & 44 & 29.3 \\
\hline & None & 2 & 1.3 \\
\hline
\end{tabular}

\subsection{Participants' clinical characteristics}

Participants' clinical information was categorized and analyzed using descriptive statistics. The most common duration of CKD was 13-36 months (44.7\%) and the most common duration of hemodialysis treatment was 13-36 months (43.3\%). More than half of the participants had both diabetes and hypertension as comorbidities. The details are provided in Table 2.

Table 2: Participants' clinical characteristics 


\begin{tabular}{lccc}
\hline Variables & Frequency (n) & Percentage (\%) \\
\hline Duration of chronic kidney disease (months) & $1-12$ & 33 & 22.0 \\
& $13-36$ & 67 & 44.7 \\
& $37-60$ & 35 & 23.3 \\
Duration of hemodialysis treatment (months) & $61-300$ & 15 & 10.0 \\
& $13-36$ & 53 & 35.3 \\
Comorbidity & $37-60$ & 23 & 43.3 \\
Diabetes & $61-100$ & 09 & 15.3 \\
Hypertension & & 06 & 6.0 \\
Diabetes and hypertension & 38 & 4.0 \\
None & & 95 & 25.3 \\
\hline
\end{tabular}

\subsection{Individual factors associated with adherence to dietary prescription}

The individual factors investigated in this study were socio-demographic characteristics, family support (including family awareness and food preparation), and clinical characteristics. Adherence to dietary prescription was significantly affected by age $\left(\chi^{2}=21.138, d f=2, p=0.000\right)$, permanent residence $\left(\chi^{2}=5.586, d f=1, p=0.018\right)$, employment status $\left(\chi^{2}=10.160, d f=3, p=0.017\right)$, duration of $\operatorname{CKD}\left(\chi^{2}=15.583, d f=3, p=0.001\right)$, comorbidity $\left(\chi^{2}=8.988, d f=3, p=0.029\right)$, and family awareness $\left(\chi^{2}=8.556, d f=1, p=0.003\right)$. Other variables are shown in Table 3.

Table 3: Individual factors associated with adherence to dietary prescription among Patients with chronic kidney disease on hemodialysis. 


\section{Adhered Not} adhered

Age (years)

$$
\text { 18-37 }
$$

38-57

58-77

Permanent residence

Dar es Salaam

Upcountry

Gender

Male

Female

Marital status

Single

Married

Cohabiting

Divorce

Widow

Educational level

Primary

Secondary

Collage/university

None

Employment status

Employed

Self employed

Retired

None

Duration of chronic kidney disease (months)

$1-12$
$13-36$
$37-60$
$61-300$

13

63

1

3

6

27

33

25

1

$\begin{array}{ll}12 & 24 \\ 38 & 33 \\ 36 & 07\end{array}$

63

23

35

29

63

23

40

24

33

$$
\text { (1) }
$$

$21.138 \quad 2$

$0.000 *$

(1)


Duration of hemodialysis treatment (months)

1-12

$13-36$

$37-60$

61-300
35

37

12

02

02

29

55

Three time a week

Comorbidity

Hypertension

Diabetes and hypertension

Family awareness
81

05
Attendance to hemodialysis schedule
Diabetes

None

Yes

No

Once a week

No
02

48

29

07

07

01

$1.170 \quad 2$

0.557 27

36

04

$8.988 \quad 3$

0.029* 47

09

04

\subsection{Associations between healthcare system-related factors and adherence to dietary prescription}

The healthcare system-related factors investigated in this study were: sources of nutritional information; attendance of nutrition counseling; affordability, payment, and convenience of nutritional counseling services; and conversation with a healthcare provider on dietary adherence. Dietary adherence was significantly associated with affordability $\left(\chi^{2}=4.066\right.$, $\mathrm{df}=1, \mathrm{p}=0.044)$, convenience of nutritional counseling services $\left(\chi^{2}=7.996, \mathrm{df}=3, \mathrm{p}=0.046\right)$, and conversation with healthcare provider on nutrition counseling $\left(\chi^{2}=16.301, \mathrm{df}=6\right.$, $\mathrm{p}=0.012$ ) as shown on Table 4 below.

Table 4: Healthcare system factors associated with adherence to dietary Prescription among patients with chronic kidney disease on hemodialysis 


\begin{tabular}{|c|c|c|c|c|c|}
\hline \multirow[t]{2}{*}{ Variables } & \multicolumn{2}{|c|}{$\begin{array}{l}\text { Participants } \\
\text { (n) }\end{array}$} & \multirow[t]{2}{*}{$\chi^{2}$} & \multirow[t]{2}{*}{$\mathrm{Df}$} & \multirow[t]{2}{*}{$\begin{array}{l}\mathrm{P}- \\
\text { value }\end{array}$} \\
\hline & Adhered & $\begin{array}{l}\text { Not } \\
\text { adhere }\end{array}$ & & & \\
\hline Source of nutritional information & & & 3.041 & 6 & 0.804 \\
\hline Doctor & 19 & 15 & & & \\
\hline Nurse & 06 & 02 & & & \\
\hline Nutritionist & 42 & 31 & & & \\
\hline Family members & 11 & 08 & & & \\
\hline Fellow patient & 06 & 04 & & & \\
\hline Friends & 0 & 01 & & & \\
\hline Media & 02 & 03 & & & \\
\hline
\end{tabular}

Affordability of nutritional counseling services

\begin{tabular}{llllll} 
Yes & 62 & 36 & 4.066 & 1 & $\mathbf{0 . 0 4 4}^{*}$ \\
No & 24 & 28 & & & \\
\hline
\end{tabular}

Source of payment for nutritional counseling service

$\begin{array}{llllll}\text { Self } & 16 & 18 & 2.260 & 2 & 0.323 \\ \text { Health insurance } & 36 & 21 & & & \\ \text { Free service } & 34 & 25 & & & \end{array}$

Convenience of nutritional counseling service.

Yes

No because I have to come to early or late

No, because nutritionist do not have

clear schedule

No, because it differs with dialysis

schedule

Conversation with healthcare provider on

dietary recommendation

This week

Last week

One month ago

More than 1 month ago

When I started hemodialysis for the first 29

1

06

16

23

14

12

26
14

34

14

$7.996 \quad 3$

09
$0.046 *$

08

33

time 


\subsection{Regression analysis of factors associated with adherence to dietary prescription among patients with CKD on hemodialysis.}

Variables that were significant in the chi-square tests were analyzed using logistic regression to evaluate if they predicted the likelihood of adherence among participants. As presented in Table 5, dietary adherence was significantly and independently affected by age $(p=0.002)$, duration of CKD $(p=0.002)$, permanent residence $(p=0.011)$, and conversation with a healthcare provider about recommended diet $(p=0.034)$.

Table 5: Predictors of adherence to dietary prescription

\begin{tabular}{lllllll}
\hline Predictor Variables & B & S.E & Wald & Df & Sig. & Exp(B) \\
\hline & & & & & & 0.369 \\
Age of participant & -0.997 & 0.327 & 9.283 & 1 & .002 & \\
Permanent residence & 1.144 & 0.448 & 6.514 & 1 & .011 & 3.138 \\
\hline Employment status & 0.075 & 0.173 & 0.189 & 1 & .664 & 1.078 \\
\hline Duration of chronic kidney disease & 0.822 & 0.266 & 9.586 & 1 & .002 & 2.276 \\
\hline Comorbidity of illness & -0.569 & 0.332 & 2.933 & 1 & .087 & 0.566 \\
\hline $\begin{array}{l}\text { Family awareness on dietary } \\
\text { recommendation }\end{array}$ & & & & & & \\
Affordability of nutritional counseling & 0.312 & 0.701 & 3.506 & 1 & .061 & 3.715 \\
\hline Convenience nutritional counseling schedule & 0.163 & 0.173 & 0.878 & 1 & .349 & 1.176 \\
\hline $\begin{array}{l}\text { Conversation with healthcare provider on } \\
\text { dietary recommendation }\end{array}$ & & & & & & \\
\hline Constant & -3.432 & 0.204 & 4.485 & 1 & .034 & 1.541 \\
\hline
\end{tabular}

\section{Discussion}

This study investigated determinants of adherence to dietary prescription among patients with ESRD undergoing hemodialysis in Dar es Salaam, Tanzania. This is the first study conducted in the Tanzanian context to investigate factors associated with dietary adherence among this population. Determinants of adherence to dietary prescription included age, employment status, comorbidity, duration of CKD, family awareness, affordability and convenience of nutritional counseling services, and conversation with a 
healthcare provider regarding dietary recommendations. Predictors of dietary adherence were age, residence, duration of CKD, and conversation with a healthcare provider about recommended diet $(13,14,21-23)$. Developing understanding of these determinants is important to inform development of strategies for effective nutritional counseling to improve adherence to dietary prescription.

Participants' age was significantly associated with adherence to dietary prescription. Older patients were more likely to adhere than younger patients. Other studies suggested that young adults reported facing difficulties complying with dietary advice (21). In addition, unavailability of the recommended foods may result in non-adherence, as patients tend to consume what is available and accessible in their community (14). Our finding revealed that adherence to dietary prescription was significantly affected by permanent residence; therefore we speculated that food diversity might be affected by location of individual residence.

Being unemployed increases the state of poverty, which may result in non-adherence to dietary recommendations among patients with CKD (24). This suggests that having a reliable income may facilitate adherence because of the ability to purchase the recommended foods. However, a previous study (13) reported that patients who were not working were more likely to adhere to a renal diet than employed patients.

Duration of CKD was associated with adherence to dietary prescription among our participants. Patients with short duration of illness tended to adhere to dietary recommendations more than those with a long duration of illness. A previous study suggested that patients with long duration of CKD may encounter challenges in managing a complex diet that they are required to practice for years (25). In addition, patients with comorbidity (e.g., diabetes) faced more difficulties in complying with diet and treatment compared with those without comorbidity (26). This was consistent with our finding that comorbidity was associated with adherence to dietary prescription. However, another study (14) reported no association between comorbidity and adherence to dietary prescription. We speculated that differences in the study settings and cultural diversity might have influenced the discrepancy in these results.

We also found a significant relationship between family support and adherence to dietary prescription. Patients who had family support tend to be more likely to adhere to dietary prescription than those without family support (27). Moreover, the person involved in food preparation needs sufficient knowledge about food composition and its complexity in preparation (22). From our finding we speculate that, family member being aware of dietary recommendation and having the knowledge on food preparation increases the likelihood of adherence to patients.

Affordability of nutritional counseling was associated with adherence to dietary prescription among our participants. This was consistent with previous reports that indicated patients who were able to attend nutritional counseling were more likely to adhere to dietary prescription (23), whereas lower family income increased the risk for non-adherence (28). These results suggested that the ability of a patient to pay for nutritional counseling services could impact dietary adherence. 
Similarly, patients who had conversations with nutritionists and received frequent sensitization on dietary issues from health workers adhered to dietary recommendations more than those who did not have such conversations (29). In our study, convenience of nutritional counseling services and conversations with a healthcare provider on dietary recommendation were significantly associated with adherence to dietary prescriptions. We believe that knowledge and sensitization on why a prescribed diet is necessary may influence dietary adherence.

Age, permanent residence, duration of CKD, and conversation with a healthcare provider on dietary recommendations were identified as predictors of adherence to dietary prescription in this study. A previous study (21) reported that adult patients perceived themselves as more vulnerable to poor health outcomes, and therefore tended to comply with medical instructions. Our finding that patients with short duration of CKD were more likely to adhere to dietary recommendations than those with long duration may be because patients experience difficulty complying with the same diet for years (25). Furthermore, participants' permanent residence and conversation with their healthcare provider on dietary recommendations predicted the likelihood of adherence. This may be because available food variety is linked with individual residence, and nutritional education is considered effective in increasing patient adherence to dietary recommendations (14).

\section{Study limitation}

This study has some limitations, the study was conducted at tertiary hospital (Muhimbili National Hospital); therefore, the findings may differ from those in other settings. However, Muhimbili Hospital may be representative of different locations around Tanzania as it is a national referral hospital. In addition, this study used a cross sectional design, and does not provide evidence to establish causeeffect relationships. However, we revealed factors associated with dietary adherence among patients with CKD undergoing hemodialysis. This study required participants to report adherence behavior that occurred in the past, and might have led to recall bias. To minimize this issue, we used a questionnaire with items that explored easily memorable, general aspects of adherence. Moreover, we did not investigate adherence to specific dietary recommendations (e.g., low protein, phosphorus, and potassium); therefore, the findings showed general adherence to any dietary advice given to a participant by their healthcare providers.

\section{Conclusion}

This study demonstrated determinants of adherence to dietary prescription among patients with ESRD in Tanzania. Almost half of the study participants did not adhere to their prescribed diet, which highlights the need for interventions focused on the identified determinants to improve adherence to dietary prescription. This can be achieved by understanding barriers and modifying the healthcare delivery system to meet individual patient needs. Further research should be conducted to explore knowledge 
among patients with ESRD regarding dietary recommendations. Use of qualitative or mixed method designs will offer an in-depth understanding on the determinants of adherence to dietary prescription.

\section{Practical implication.}

Patient in hemodialysis requires dietary modification to reduce severity of morbidity and mortality. This study highlights the key factors associated with adherence to dietary prescription among patient with end stage renal disease. Also, it provides information on the role of adherence to nutritional therapy in managing ESRD. Furthermore adds knowledge to healthcare providers on predictive factors toward dietary adherence among patient with ESRD.

\section{Policy implication}

Provide information to policy maker on dietary adherence and related strategies on development or review existing policy on management of CKD. The current study highlights the key areas on dietary adherence that healthcare provider can emphasize on provision of nutritional management on patient with CKD.

\section{Abbreviations}

CKD: chronic kidney disease, ESRD: end-stage renal disease.

\section{Declarations}

\section{Ethical Considerations}

Aga Khan University Ethical Review Committee approved this study. Permission to conduct the study was obtained from the management of Muhimbili National Hospital. Written informed consent was obtained from each participant before the study started. Consent forms were written in Kiswahili to ensure participants fully understood. The consent form included an explanation of the purpose of the study, confidentially of the information, and the right to withdraw at any point.

\section{Consent for publication}

Not applicable.

\section{Availability of data and materials}

The dataset generated and/or analyzed during the present study is available from the corresponding author upon reasonable request. Permission to access this data will be required from the Aga Khan University.

\section{Competing interest}


The authors declare that they have no competing interests.

\section{Funding}

None.

\section{Authors' contributions}

$\mathrm{RB}, \mathrm{ML}$, and TM contributed to the conception and design of the study. RB was involved in the fieldwork of data collection. TM and RB performed data analysis and interpretation. RB drafted the manuscript, and $M L$ and TM critically reviewed the manuscript for important intellectual content. All authors read and approved the final manuscript.

\section{Acknowledgements}

We would like to thank all patients who agreed to participate in this study, Muhimbili National Hospital for granting permission to conduct the study, and the research assistants for their outstanding commitment during data collection.

\section{References}

1. Nazar CMJ. Significance of diet in chronic kidney disease. Journal of nephropharmacology. 2013;2(2):37.

2. Xie Y, Bowe B, Mokdad AH, Xian H, Yan Y, Li T, et al. Analysis of the Global Burden of Disease study highlights the global, regional, and national trends of chronic kidney disease epidemiology from 1990 to 2016. Kidney international. 2018;94(3):567-81.

3. ElHafeez SA, Bolignano D, D’Arrigo G, Dounousi E, Tripepi G, Zoccali C. Prevalence and burden of chronic kidney disease among the general population and high-risk groups in Africa: a systematic review. BMJ open. 2018;8(1):e015069.

4. Hodel NC, Hamad A, Praehauser C, Mwangoka G, Kasella IM, Reither K, et al. The epidemiology of chronic kidney disease and the association with non-communicable and communicable disorders in a population of sub-Saharan Africa. PloS one. 2018;13(10):e0205326.

5. Kaze AD, llori T, Jaar BG, Echouffo-Tcheugui JB. Burden of chronic kidney disease on the African continent: a systematic review and meta-analysis. BMC nephrology. 2018;19(1):125.

6. Ploth DW, Mbwambo JK, Fonner VA, Horowitz B, Zager P, Schrader R, et al. Prevalence of CKD, diabetes, and hypertension in rural Tanzania. Kidney international reports. 2018;3(4):905-15.

7. Stanifer JW, Maro, V., Egger, J., Karia, F., Thielman, N., Turner, E. L., ... \& Patel, U. D. The epidemiology of chronic kidney disease in Northern Tanzania: a population-based survey. PloS one. 2015;10:4. 
8. Saran R, Bragg-Gresham JL, Rayner HC, Goodkin DA, Keen ML, Van Dijk PC, et al. Nonadherence in hemodialysis: associations with mortality, hospitalization, and practice patterns in the DOPPS. Kidney international. 2003;64(1):254-62.

9. Herselman M. Non-adherence to dietary prescriptions in chronic kidney disease. South African Journal of Clinical Nutrition. 2008;21(2):13-4.

10. Rysz J, Franczyk B, Ciałkowska-Rysz A, Gluba-Brzózka A. The effect of diet on the survival of patients with chronic kidney disease. Nutrients. 2017;9(5):495.

11. Denhaerynck K, Manhaeve D, Dobbels F, Garzoni D, Nolte C, De Geest S. Prevalence and consequences of nonadherence to hemodialysis regimens. American Journal of Critical Care. 2007;16(3):222-35.

12. Luis D, Zlatkis K, Comenge B, García Z, Navarro JF, Lorenzo V, et al. Dietary quality and adherence to dietary recommendations in patients undergoing hemodialysis. Journal of Renal Nutrition. 2016;26(3):190-5.

13. Lambert K, Mullan J, Mansfield K. An integrative review of the methodology and findings regarding dietary adherence in end stage kidney disease. BMC nephrology. 2017;18(1):318.

14. Opiyo RO, Nyasulu PS, Olenja J, Zunza M, Nguyen KA, Bukania Z, et al. Factors associated with adherence to dietary prescription among adult patients with chronic kidney disease on hemodialysis in national referral hospitals in Kenya: a mixed-methods survey. Renal Replacement Therapy. 2019;5(1):41.

15. Kayombo EJ, Uiso FC, Mahunnah RL. Experience on healthcare utilization in seven administrative regions of Tanzania. Journal of ethnobiology and ethnomedicine. 2012;8(1):1-8.

16. Stanifer JW, Patel UD, Karia F, Thielman N, Maro V, Shimbi D, et al. The determinants of traditional medicine use in northern Tanzania: a mixed-methods study. PloS one. 2015;10(4):e0122638.

17. Furia FF, Shoo J, Ruggajo PJ, Kilonzo K, Basu G, Yeates K, et al. Developing nephrology services in low income countries: a case of Tanzania. BMC nephrology. 2019;20(1):378.

18. Alikari V, Tsironi M, Matziou V, Tzavella F, Stathoulis J, Babatsikou F, et al. The impact of education on knowledge, adherence and quality of life among patients on haemodialysis. Quality of Life Research. 2019;28(1):73-83.

19. Israel GD. Determining sample size. 1992.

20. Kim Y, Evangelista LS, Phillips LR, Pavlish C, Kopple JD. The End-Stage Renal Disease Adherence Questionnaire (ESRD-AQ): testing the psychometric properties in patients receiving in-center hemodialysis. Nephrology nursing journal: journal of the American Nephrology Nurses' Association. 2010;37(4):377. 
21. Clark-Cutaia MN, Ren D, Hoffman LA, Burke LE, Sevick MA. Adherence to hemodialysis dietary sodium recommendations: influence of patient characteristics, self-efficacy, and perceived barriers. Journal of Renal Nutrition. 2014;24(2):92-9.

22. Chan YM, Zalilah MS, Hii SZ. Determinants of compliance behaviours among patients undergoing hemodialysis in Malaysia. PloS one. 2012;7(8):e41362.

23. Paes-Barreto JG, Silva MIB, Qureshi AR, Bregman R, Cervante VF, Carrero JJ, et al. Can renal nutrition education improve adherence to a low-protein diet in patients with stages 3 to 5 chronic kidney disease? Journal of Renal Nutrition. 2013;23(3):164-71.

24. Chironda G, Bhengu B. Contributing factors to non-adherence among chronic kidney disease (CKD) patients: a systematic review of literature. Medical \& Clinical Reviews. 2016;2(4):29.

25. Lindberg M. Excessive Fluid Overload among Haemodialysis Patients: Prevalence, Individual Characteristics and Self-regulation of Fluid Intake. 2010.

26. Shirazian S, Crnosija N, Weinger K, Jacobson AM, Park J, Tanenbaum ML, et al. The selfmanagement experience of patients with type 2 diabetes and chronic kidney disease: a qualitative study. Chronic illness. 2016;12(1):18-28.

27. Yokoyama Y, Suzukamo Y, Hotta O, Yamazaki S, Kawaguchi T, Hasegawa T, et al. Dialysis staff encouragement and fluid control adherence in patients on hemodialysis. Nephrol Nurs J. 2009;36(3):28997.

28. Pang SK, Ip WY, Chang AM. Psychosocial correlates of fluid compliance among Chinese haemodialysis patients. Journal of Advanced Nursing. 2001;35(5):691-8.

29. Ford JC, Pope JF, Hunt AE, Gerald B. The effect of diet education on the laboratory values and knowledge of hemodialysis patients with hyperphosphatemia. Journal of Renal Nutrition. 2004;14(1):3644. 